\title{
Methode zur Einflussanalyse in der Szenario- Technik auf Basis gerichteter Graphen
}

Iris Gräßler ${ }^{1}$, Henrik Thiele ${ }^{1}$, Philipp Scholle ${ }^{1}$

${ }^{1}$ Research Group for Product Creation, Heinz Nixdorf Institute, Paderborn University, Germany

\section{Abstract}

In an environment of increasing market dynamics and shortening product lifecycles, strategic foresight with scenario-technique is a way for the anticipation of future market and technological developments to identify innovations. Within scenario-technique, influence analysis has a broad impact on both efforts to build scenarios and quality of such scenarios. To reduce the complexity of scenario-building, a set of key influence factors is selected. In this paper, a novel approach based on a modified page-rank algorithm for the selection of key influence factors is presented. By the holistic approach, direct and indirect influences on factors are considered, in relation to their adjacent nodes on the representing graph. Furthermore, the robustness of the selection is improved.

Keywords: Scenario-Technique, Page-Rank, Strategic Planning, Foresight

\section{Einleitung und Motivation}

In Zeiten zunehmend dynamischer Märkte und verkürzter Produktlebenszyklen besteht der Bedarf einer treffsicheren Zukunftsvorausschau sowohl zur zielgerichteten Entwicklung neuer Produkte als auch zur Positionierung als Unternehmen. Sollen zukünftige Entwicklungen antizipiert werden, müssen relevante Einflussfaktoren identifiziert werden. Durch Trends wie Digitalisierung 
und Globalisierung steigt neben der Anzahl von Einflussfaktoren auch die Komplexität der Betrachtung. Insbesondere im Kontext der Szenario-Technik als Werkzeug der Zukunftsvorausschau bildet die Einflussanalyse den Schlüssel zur Entwicklung von Szenarien. Ziel der Szenario-Technik ist dabei die Identifikation zukünftiger Innovationen durch die Antizipation von Kunden- oder Marktbedürfnissen, aber auch Technologieentwicklungen [1]. Des Weiteren können mittels Szenario-Technik mögliche Restriktionen im Design for X (DfX) wie die Veränderung fertigungstechnischer Restriktionen oder Änderungen der Anforderungen entlang des Produktentstehungsprozesses antizipiert werden $[2,3]$.

\section{Stand der Forschung}

Ausgehend vom Szenario-Begriff der militärischen Planung (siehe hierzu [4]) sind im strategischen Management verschiedene Schulen und Prozessmodelle der Szenario-Technik entwickelt worden [5]. Übergeordnetes Ziel ist dabei das Antizipieren von zukünftigen Entwicklungen oder Zukunftsbildern („Szenarien") für einen Betrachtungsgegenstand. Dieser Szenario-Begriff muss dabei vom Begriff der Anwendungsszenarien eines Produktes unterschieden werden.

\subsection{Schulen innerhalb der Szenario-Technilk}

Innerhalb der intuitiven Schule der Szenario-Technik erfolgt die Bildung der Szenarien auf Basis eines diskursiven, nicht formalisierten Prozesses innerhalb einer Gruppe von Experten [6, 7]. Dabei werden keine mathematischen Modelle verwendet. Die Szenarien werden mit Ansätzen der Cross-Impact Schule gebildet. Sie basieren auf der Analyse von Wechselwirkungen von Einflussfaktoren [8]. Für diese werden die Entwicklungsrichtungen („Projektionen") der Einflussfaktoren identifiziert, für welche Eintrittswahrscheinlichkeiten geschätzt werden. Die Szenarien werden dann über die Kombination der verschiedenen Projektionen miteinander und die Berechnung angepasster bedingter Wahrscheinlichkeiten gebildet [8]. Die Schätzung der Wahrscheinlichkeiten ist hierbei eine Hauptfehlerquelle $[6,9]$. Innerhalb der konsistenzbasierten Schule erfolgt die Bildung der Szenarien nicht auf Basis von Wahrscheinlichkeiten, sondern über die Bewertung der Konsistenz der Projektionen. Hierzu sind in der Vergangenheit verschiedene Vorgehensmodelle entstanden (siehe hierzu [10-12]).

\subsection{Methoden zur Einflussanalyse als Teil eines agilen Vorgehens zur strategischen Planung}

Durch Gräßler et al. wurde das agile Vorgehensmodell der strategischen Planung publiziert (siehe Abbildung 1) [13]. 


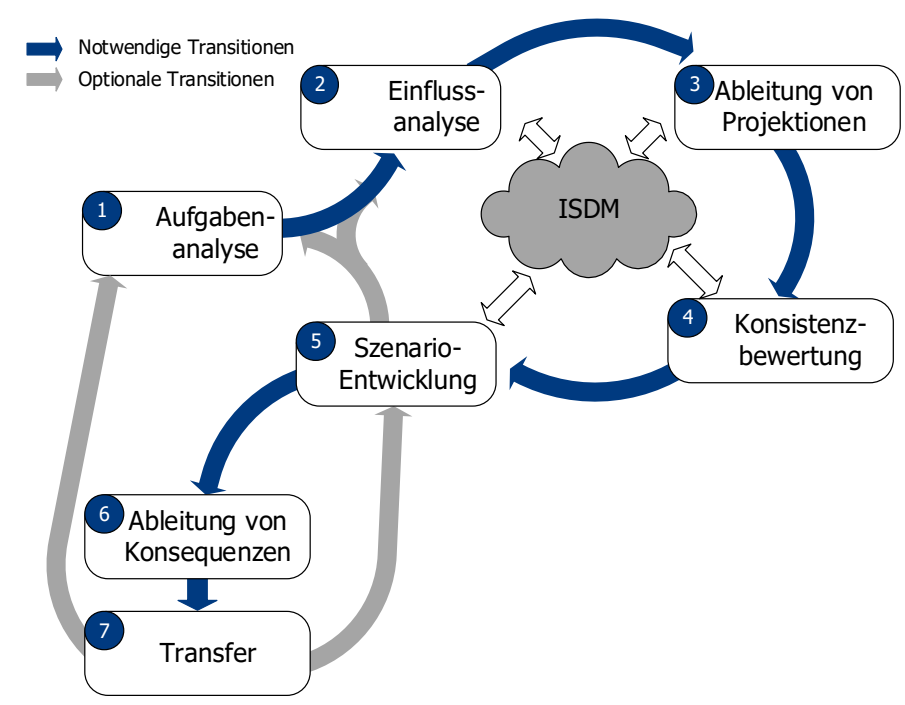

Abbildung 1: Agiles Vorgehensmodell zur strategischen Planung [13]

Aufbauend auf der Zielsetzung der Szenario-Entwicklung (Phase 1) werden im zweiten Schritt („Einflussanalyse") die Einflussfaktoren gesammelt, welche zukünftige Entwicklungen des Betrachtungsgegenstandes beeinflussen. Für diese werden die Wechselwirkungen in einer Einflussmatrix bewertet. Hierbei erfolgt eine Differenzierung zwischen verschiedenen Einflussstärken. Aufgrund des hohen Aufwandes der folgenden Schritte („Ableitung von Projektionen" und "Konsistenzbewertung") wird im Folgenden die Anzahl der Einflussfaktoren reduziert. Die Auswahl der Schlüsselfaktoren erfolgt durch Betrachtung der Aktivität und Passivität der Einflussfaktoren, welche sich aus der Einflussmatrix ergibt [10]. Durch Reibnitz [10] wird dabei das System-Grid (Abbildung 2) verwendet, welches die Einflussfaktoren in die vier Kategorien aktive, ambivalente, passive und nicht-ambivalente Systemelemente kategorisiert. Dabei erfolgt noch keine Erfassung der möglichen indirekten Einflüsse höherer Ordnung.

Durch Gausemeier wird die Einflussanalyse um die Kriterien Dynamik und Impulsivität der Einflussfaktoren erweitert, welche aus der Aktiv- und Passivsumme berechnet werden [12]. Auch hierbei erfolgt die Auswahl auf Basis heuristischer Selektionsregeln. Zur Betrachtung komplexerer Zusammenhänge werden Methoden der Social Network Analysis durch Grienitz und Schmidt für die Einflussanalyse verwendet [14]. Hierbei wird die Einflussmatrix als gerichteter Graph aufgefasst. Die Kriterien Zentralität, Dichte und Kohäsion werden dann zur Auswahl der Schlüsselfaktoren herangezogen [14]. In diesem Ansatz bleiben die indirekten Einflüsse höherer Ordnung unberücksichtigt. 
Zur Identifikation der indirekten Einflüsse höherer Ordnung werden durch Gausemeier et al. heuristische Dämpfungsfaktoren eingeführt. Die einzelnen Einflüsse höherer Ordnung werden auf die direkten (in der Einflussmatrix dargestellten) Einflüsse hinzuaddiert [12]. Die Wahl der Dämpfungsfaktoren ist hierbei heuristisch und erfolgt durch den Nutzer. In der MICMAC-Methode nach Godet werden die Einflüsse höherer Ordnung durch die Multiplikation binärer Einflussmatrizen identifiziert [15]. Die Aussage über die Stärke der Einflüsse geht dabei verloren. Das Abbruchkriterium des Algorithmus ist hierbei eine Stabilität der sich aus der Betrachtung der Multiplikation der binären Einflussmatrix mit sich selbst ergebenen Reihenfolge [15]. Das Abbruchkriterium wird dabei durch den Nutzer vorgegeben.

Auf Basis der ausgewählten Schlüsselfaktoren werden anschließend für diese Faktoren Projektionen gebildet, welche in Phase 4 paarweise auf Konsistenz bewertet werden. Die Konsistenz ist hierbei als Widerspruchsfreiheit eines gemeinsamen Auftretens zweier Projektionen in einem Szenario definiert (siehe hierzu [16]). Zur Bildung der Szenarien in Phase 5 können dann neben einer Bildung aller möglichen Szenarien (Vollenumeration) auch Ansätze zur Teilenumeration wie Branch-and-Bound Algorithmen, lineare Optimierung oder evolutionäre Algorithmen verwendet werden [17-19]. Hierbei werden nicht alle möglichen, sondern lediglich die Szenarien mit hoher Konsistenz gebildet. Aus der Menge der Szenarien werden dadurch wenige Szenarien selektiert, welche in den folgenden Phasen 6 und 7 analysiert und transferiert werden.

Innerhalb des agilen Vorgehensmodell müssen in einem ersten Durchlauf alle Schritte sequentiell durchlaufen werden, wobei die Annahmen laufend angepasst werden können (Notwendige und optionale Transitionen in Abbildung 1). Das agile Vorgehensmodell wird dabei vom Integrierten Szenario Datenmodell (ISDM) flankiert, in welchem alle relevanten projektübergreifenden Einflussfaktoren, Projektionen und Selektionsregeln hinterlegt sind (siehe hierzu $[20,21]$. Neben den projektübergreifenden Einflussfaktoren können auch projektspezifische Einflussfaktoren durch den Nutzer hinzugefügt werden. Diese werden dann im ISDM ergänzt und stehen in folgenden Projekten ebenfalls als Wissensbasis zur Verfügung.

\subsection{Kritik an den Methoden zur Einflussanalyse}

Ziel der dargestellten Methoden zur Einflussanalyse ist es, diejenigen Einflussfaktoren zuerst zu identifizieren, welche einen niedrigen eingehenden und einen hohen ausgehenden Impuls auf das System haben und zuletzt solche 
Faktoren, deren eingehender und ausgehender Impuls niedrig ist (vgl. Abbildung 2). Das System wird dabei als gerichteter, gewichteter Graph mit den Einflussfaktoren als Knoten beschrieben.

Aus dieser Aufgabenstellung heraus ergeben sich verschiedene Probleme. So muss bei der Berechnung des Impulses der Wert der direkt verknüpften Knoten im Sinne des Systemdenkens im globalen Kontext berücksichtigt werden, während klassische Methoden Nachbarschaftsbeziehungen nur auf lokale Weise betrachten [15]. Dies führt zu einer Unterbewertung und damit Fehlbewertung von Einflussfaktoren, insbesondere der dynamischen Treiber, in Bezug auf das Gesamtsystem. Die Auswahlreihenfolge der Einflussfaktoren ist nach klassischer Logik zudem instabil, da sich die Gruppe der zuerst und zuletzt zu wählenden Einflussfaktoren lediglich in der Aktivsumme unterscheidet. Eine geringe Änderung der Datengrundlage führt so zu einer diametral anderen Einordnung einzelner Einflussfaktoren. Mathematisch zeigt sich dies durch eine Unstetigkeit an der Phasengrenze zwischen dem aktiven und puffernden Feld im System-Grid. Somit ist die Priorisierung nicht robust gegenüber Änderungen. Gleichzeitig ist die Auswahl der Schlüsselfaktoren oftmals von heuristischen Annahmen abhängig. Dieses erforderliche Expertenwissen ist hierbei kritisch für die Ergebnisgüte des gesamten Szenario-Prozesses [6, 7]. Gleichzeitig ist der hohe Aufwand für die Durchführung der Szenario-Technik einer der Hauptkritikpunkte an der Szenario-Technik im Allgemeinen [6, 22, 23].

\section{Forschungsziel und Vorgehensweise}

Für die Entwicklung einer holistischen Methode zur Auswahl von Einflussfaktoren der Gestaltung von Zukunftsszenarien folgt demnach die folgende Forschungsfrage: Wie können die relevanten (Schlüssel-) Einflussfaktoren unter Berücksichtigung der Dynamik des Systems robust identifiziert werden?

Zur Strukturierung des methodischen Vorgehens wurde die Design Research Methodology angewendet [24]. Mit dem Forschungstyp 3 wurde ein Vorgehen gewählt, welches die umfassende Entwicklung und die initiale Validierung einer Methode ermöglicht. Zur Research Clarification wurde eine Literaturstudie durchgeführt (Abschnitt 2). Die Deskriptive Studie I erfolgt auf Basis einem Fallbeispiel aus dem EU H2020 geförderten Projekt "ANYWHERE". Dabei wurden Datensätze zur strategischen Planung innovativer Dienstleistungen zur Vorhersage und Auswirkungsanalyse bei Extremwetterereignissen analysiert (siehe hierzu auch [21]). Das System-Grid nach Reibnitz mit den Einflussfaktoren aus Tabelle 1 ist in Abbildung 2 dargestellt. Hierbei bestätigt sich die in Abschnitt 2.3 beschriebene Problematik der Robustheit an Einflussfaktor E8 (Auswirkungen von Extremwetterereignissen auf dem Güterverkehr). 
Durch eine marginale Änderung wird dieser Einflussfaktor signifikant anders eingeordnet. An Einflussfaktor E8 (Jahresmitteltemperatur) wird deutlich, dass die MICMAC Methode die Dynamik des Systems unzureichend modelliert, da diese eindeutige Treibergröße von Extremwetterereignissen deutlich unterbewertet ist.

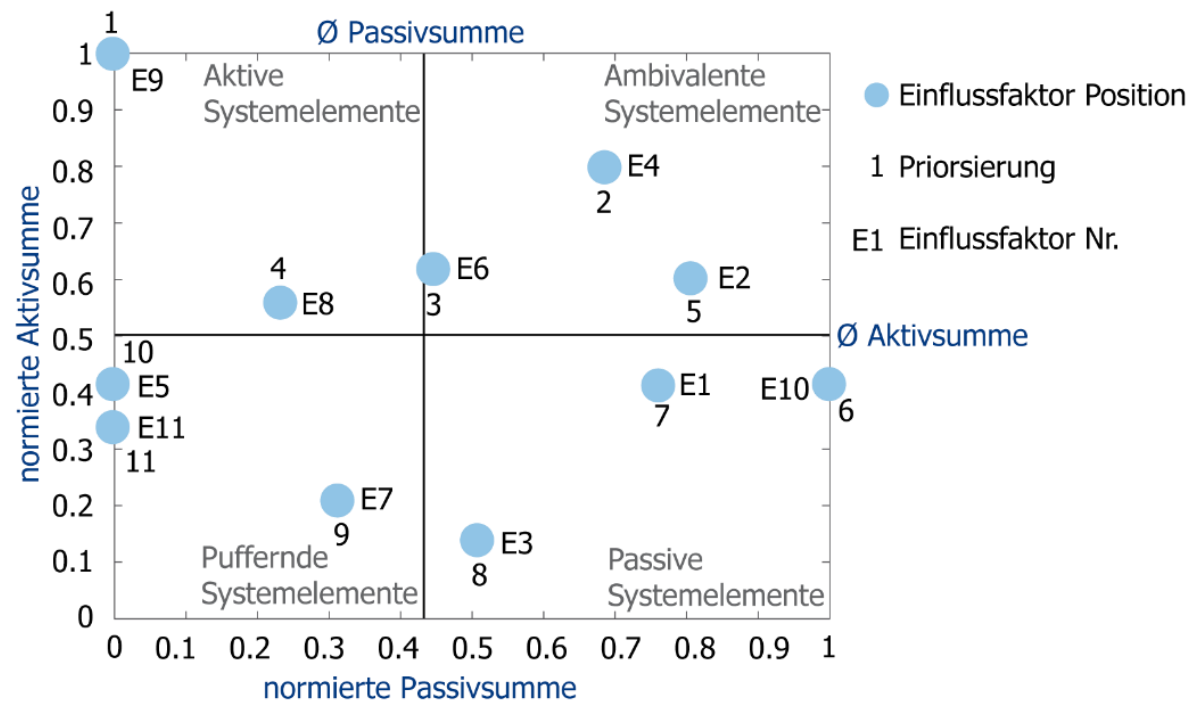

Abbildung 2: System-Grid nach Anwendung der MICMAC-Methode

Darauf aufbauend erfolgt in der Präskriptiven Studie die Entwicklung der Methode zur Einflussanalyse in der Szenario-Technik (Abschnitt 4). Die Validierung auf Basis eines Fallbeispiels (Deskriptive Studie II) wird in Abschnitt 5 beschrieben.

\section{Methode zur Einflussanalyse in der Szenario-Technik auf Basis ge- richteter Graphen}

Durch eine Analogiebetrachtung wurde der Algorithmus identifiziert, der der entwickelten Methode zu Grunde liegt: Das World Wide Web wird durch Suchmaschinen als gerichteter Graph aufgefasst, indem jede Website einen Knoten und die Kanten des Graphen einen Hyperlink von einer Website auf eine weitere darstellen; dieser Interpretation folgend wurde durch Brin und Page der Page-Rank Algorithmus entwickelt und nachfolgend in der Suchmaschine Google implementiert [25]. 
Die Priorisierung eines Einflussfaktors durch den Page-Rank Algorithmus ergibt sich aus der Impulswirkung der anderen Knoten des Netzwerks, unter Berücksichtigung des Impulses, welchen dieser ausübt. Die Modellierung geschieht anhand eines Zufalls-Surfers, der sich von einem Knoten ausgehend entweder mit gleicher Wahrscheinlichkeit in einen der benachbarten Knoten bewegt oder mit einer globalen Wahrscheinlichkeit $d \in[0 ; 1]$ in einen beliebigen Knoten teleportiert. Die Berechnungsvorschrift ist in der nachfolgenden Formel gegeben.

Formel 1: Berechnungsvorschrift des Page-Rank Algorithmus

$$
P R\left(e_{i}\right)=\frac{1-d}{n}+d \sum_{e_{j} \in E_{i}^{\text {in }}}\left(\sum_{r_{k} \in E_{j}^{\text {out }}} \frac{P R\left(e_{j}\right)}{v_{k j}}\right)
$$

Hier sei ein Knoten durch die Variable $e_{i}$ eindeutig benannt. Die von $e_{i}$ einund ausgehenden Kantenmengen sind durch die Mengen $E_{i}^{\text {in }}$ und $E_{i}^{\text {out }}$ gegeben und die Gewichtung einer Kante, die von $e_{i}$ auf $e_{j}$ zeigt, sei mit $v_{i j}$ gegeben, wobei $V=\left(v_{i j}\right)$ die Vernetzungsmatrix der Einflussanalyse sei. Der Page-Rank wird anhand der gegebenen Formel rekursiv berechnet, indem alle Page-Ranks $P R\left(e_{i}\right)$ der Kanten auf $\frac{1}{\#\{\text { Knoten }\}}=\frac{1}{n}$ gesetzt werden. In jedem Iterationsschritt wird das Page-Rank Gewicht $P R\left(e_{i}\right)$ auf die benachbarten Knoten gemäß des Gewichtes $v_{k j}$ verteilt. Der globale Zufallsfaktor $d$ gibt die zufällige Wahl eines nicht-benachbarten Knotens wider. Für alle Werte $d<1$ konvergiert der Algorithmus und liefert eine eindeutige Lösung [26]. Durch diese Umverteilung ergibt sich direkt, dass $\sum_{n} P R\left(e_{i}\right)=1$ in jedem Iterationsschritt ist.

Übertragen auf die Einflussanalyse ergibt sich mit $P R\left(e_{i}\right)$ der Passivwert für den Impuls eines Einflussfaktors durch die restlichen Einflussfaktoren im globalen Kontext. Durch Iteration des gesamten Systems wird jeder Impulswert stets im Verhältnis zu den restlichen Einflussfaktoren betrachtet. Als Interpretation des Zufallswertes $d$ wird die Unsicherheit in der Datenerhebung gewählt. Bei $d \approx 1$ sei jeder Wirkzusammenhang im betrachteten System bekannt und in $V$ niedergeschrieben. Je kleiner $d$, desto mehr wird zugelassen, dass ein Einflussfaktor einen Einfluss auf einen weiteren Faktor ausübt, der nicht in $V$ benannt wurde. Als Wahl des Faktors wird $d=0,85$ empfohlen [27].

Im Sinne der Einflussanalyse nach von Reibnitz ist nun ein Analogon zur Passivsumme, der Passivrang definiert. Durch Transponieren der Einflussmatrix 
$V$ lässt sich mit der entsprechend angepassten Berechnungsvorschrift ein Aktivrang $A R\left(e_{i}\right)$ definieren und berechnen. Dieser bildet das Analogon zur Aktivsumme nach von Reibnitz. Die Anwendung von Aktiv- und Passivrang im Gegensatz zur Aktiv- und Passivsumme gibt nun eine Einordnung der Einflussfaktoren im System-Grid, die die Dynamik des Gesamtsystems berücksichtigt. Der Gesamtrang eines Einflussfaktors $e_{i}$ wird durch den Vektor $r_{i} \in \mathbb{R}^{2}$ dargestellt.

Durch den holistischen Ansatz der Gesamtiteration werden insbesondere die indirekten Einflüsse beliebiger Tiefe bei der Einordnung der Einflussfaktoren berücksichtigt. Um eine eindeutige und robuste Priorisierung zu erhalten, geschieht die Auswahl der Einflussfaktoren nach einer abgewandelten SystemGrid-Logik. So werden die Einflussfaktoren über eine Ordnungszahl priorisiert. Zunächst wird ein Sternenzentrum $S$ außerhalb der Koordinaten des SystemGrid gewählt. Die Ordnungszahl berechnet sich nun als der Winkel zwischen der Vertikalen aus dem Sternenzentrum $b$ und der Geraden zwischen dem Sternenzentrum und $r_{i}$.

So ergibt sich die folgende Bildungsregel:

Formel 2: Bildungsregel der Ordnungszahl

$$
\operatorname{Ord}\left(e_{i}\right)=\arccos \left(\frac{\left(r_{i}-S\right) \cdot b}{\left|r_{i}-S\right||b|}\right)
$$

Für $\operatorname{Ord}\left(e_{i}\right)=\operatorname{Ord}\left(e_{j}\right)$ wird der Einflussfaktor zuerst gewählt, der den höheren Aktivrang besitzt. Die Ordnungszahlen genügen zudem der folgenden Regel: Die aktivsten und am wenigsten passiven Einflussfaktoren werden zuerst gewählt. Bevor ein passiver oder puffernder Einflussfaktor gewählt wird, sind alle aktiven und ambivalenten Einflussfaktoren gewählt worden. Zuletzt werden alle weder aktiven noch passiven Einflussfaktoren gewählt. Die stärkere Priorisierungsregel nach von Reibnitz genügt dieser Regel ebenso. Die Priorisierungsregel auf Basis der Ordnungszahlen ist nun robust, da $\operatorname{Ord}\left(e_{i}\right)$ als Gesamtfunktion stetig ist, während die Priorisierung nach von Reibnitz eine Unstetigkeit an der Phasengrenze zwischen dem aktiven und puffernden Bereich aufweist.

\section{Validierung}

In dem Fallbeispiel aus Abschnitt 3 ergeben sich die in Tabelle 1 dargestellten Priorisierungen der gegebenen Einflussfaktoren nach MICMAC (MM) und PageRank (PR). Der Rang wurde jeweils basierend auf Formel 2 berechnet. 
EINFLUSSFAKTOR

\begin{tabular}{l|lll} 
E01 & Eingreifen von Bund und Ländern bei Extremwetter & 7 & 4 \\
E02 & Ausgaben für den Katastrophenschutz & 5 & 6 \\
E03 & Ausfallrisiko des mobilen Internets & 8 & 9 \\
E04 & Ausfallrisiko kritischer Infrastruktur bei Extremwetter & 2 & 3 \\
E05 & Vermögensentwicklungen in Deutschland/Europa & 10 & 11 \\
E06 & Steuereinnahmen & 3 & 7 \\
E07 & Entwicklung des Gütertransports & 9 & 10 \\
E08 & Auswirkungen des Extremwetters auf den Gütertransport & 4 & 5 \\
E09 & Tatsächliche Bedrohung durch Extremwetter & 1 & 2 \\
E10 & Persönliche Vorbereitung auf Extremwetter & 6 & 8 \\
E11 & Jahresmitteltemperatur & 11 & 1
\end{tabular}

Tabelle 1: Einflussfaktoren des Fallbeispiels

Das System-Grid unter Hinzunahme der durch Page-Rank berechneten Einflüsse ist in Abbildung 3 dargestellt. Die Skalen des Page-Ranks sind so normiert, dass die MICMAC und Page-Rank Einflussfaktoren dieselben Mittelwerte besitzen. Dies stellt eine grafische Vergleichbarkeit der Werte sicher. Über die Färbung der Felder im System-Grid ist die Ordnungszahl nach Formel 2 visualisiert; die Felder nach Reibnitz durch die horizontale und vertikale auf Höhe der durchschnittlichen Aktiv- und Passivsumme.

Der zuvor als puffernd eingeordnete Einflussfaktor E11 (Jahresmitteltemperatur) wird nun korrekt als Treibergröße identifiziert und an Stelle 1 priorisiert. Der dadurch angestoßene Einflussfaktor E9 (Tatsächliche Bedrohung durch Extremwetter) wird weiterhin als wichtig (Stelle 2) identifiziert. Die Robustheit der Auswahl wird an Einflussfaktor E8 (Auswirkungen des Extremwetters auf den Gütertransport) deutlich. Eine marginale Änderung der Aktivsumme führt zur Identifizierung als pufferndes und nicht mehr aktives Element. Nach Formel 1 und Formel 2 bedeutet die marginale Änderung lediglich den Tausch der Priorisierungsreihenfolge um eine Stelle mit E2.

Dies bestätigt in einem ersten Fallbeispiel sowohl die korrekte Berücksichtigung der Dynamik eines Einflussnetzwerkes, als auch die Robustheit der Auswahl der Einflussfaktoren. Insbesondere stellt die Berücksichtigung der Einflussstärke einen Mehrwert gegenüber der MICMAC-Methode dar und die vorläufige qualitative Validierung wird als erfolgreich aufgefasst. Die Verifikation 
der auf Formel 1 basierenden Methode wurde an anderer Stelle auf quantitativer Basis geführt und zeigt die richtige Realisierung des Algorithmus gemäß der Spezifikationen der Aufgabenstellung [28].

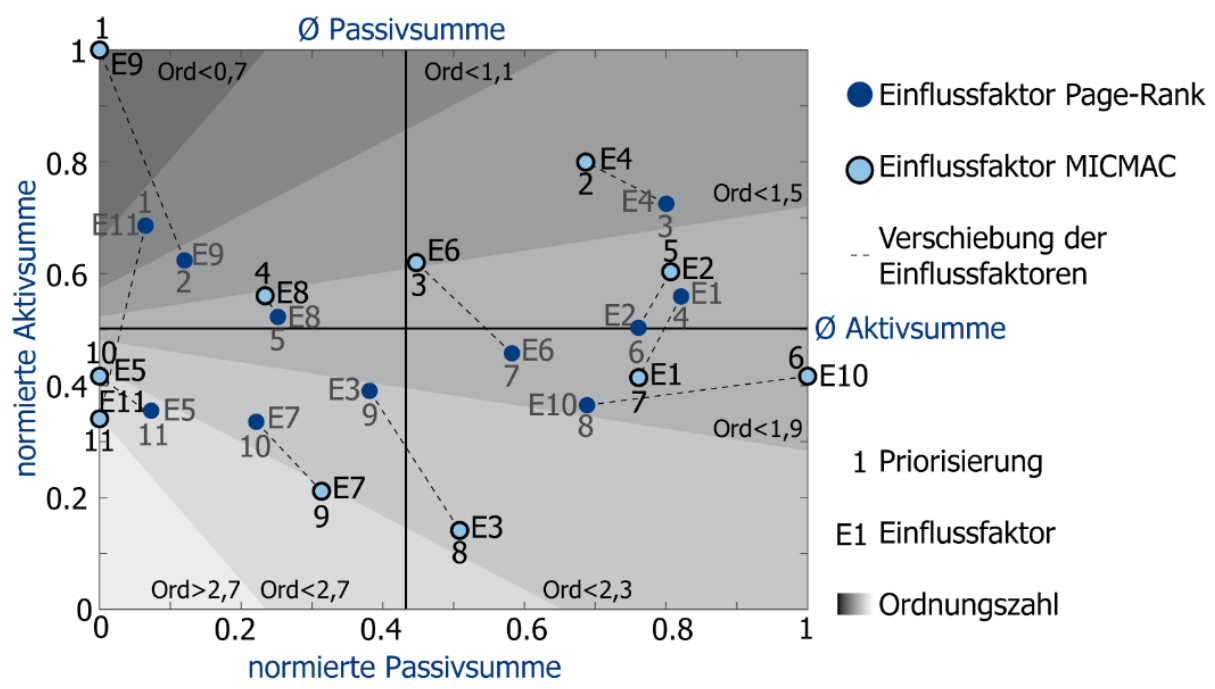

Abbildung 3: System-Grid des Fallbeispiels mit Ergebnissen des Page-Rank

\section{Zusammenfassung und Ausblick}

Die entwickelte Methode dient der Auswahl der wichtigsten Einflussfaktoren eines Innovationsprojektes in dem Graphen, der sich aus der Vernetzung zuvor identifizierter Einflussfaktoren ergibt. Die Auswahl ist eindeutig und robust. Geringfügige Änderungen in der Datengrundlage, insbesondere Berichtigungen von Nutzerfehlern, rufen somit nur geringfügige Änderungen in der Priorisierungsreihenfolge hervor. Durch die globale Betrachtung des Einflussgraphen mit Hilfe eines modifizierten Page-Rank-Algorithmus werden Faktoren korrekt als wichtig identifiziert, deren Priorisierung nach klassischen Methoden fälschlicherweise gering ist. Die so identifizierten Einflussfaktoren sind jene, die die Dynamik des Einflussnetzwerkes hervorrufen.

Die Methode ist eine Voraussetzung für die automatisierte Berechnung von Szenarien und kann so den Nutzer im Sinnes des agilen Vorgehensmodells der Szenario-Technik unterstützen [13]. Die zugrundeliegenden Algorithmen der entwickelten Methode können zudem in anderen Bereichen der Produktentwicklung zur Identifikation von Treibergrößen genutzt werden, wie zum Beispiel in der Priorisierung von Anforderungen [28]. Zudem kann in einem nächsten 
Schritt anhand historischer Daten zur Validierung eine Application Evaluation im Sinne der DRM durchgeführt werden.

\section{Literatur}

[1] Grienitz, V.: „Technologieszenarien. Eine Methodik zur Erstellung von Technologieszenarien für die strategische Technologieplanung". HNI, 2004.

[2] Gräßler, I.; Scholle, P.; Pottebaum, J.: „Integrated process and data model for applying scenario-technique in requirements engineering". In: Design Society (Hrsg.) ICED17. 21st International Conference on Engineering Design Vancouver, 2017, S. 261-270.

[3] Gräßler, I.; Scholle, P.: "Szenario-basierte Analyse von Anforderungen im Produktentwicklungsprozess". In: Krause, D.; Paetzold, K.; Wartzack, S. (Hrsg.) Beiträge zum 27. DfX-Symposium, 2016, S. 231-242.

[4] Clausewitz, C. v.: „Vom Kriege, Berlin, 1832-1834.

[5] Meyer-Schönherr, M.: "Szenario-Technik als Instrument der strategischen Planung". Verl. Wiss. und Praxis, Ludwigsburg, 1992.

[6] Mietzner, D.; Reger, G.: „Advantages and Disadvantages of Scenario Approaches for Strategic Foresight". In: International Journal Technology and Intelligence Planing 1 (2005) 2, S. 220-239.

[7] Bradfield, R.; Wright, G.; Burt, G.; Cairns, G.; Van Der Heijden, Kees: "The origins and evolution of scenario techniques in long range business planning". In: Futures 37 (2005) 8, S. 795-812.

[8] Gordon, T. J.; Hayward, H.: "Initial experiments with the cross impact matrix method of forecasting". In: Futures 1 (1968) 2, S. 100-116.

[9] Grienitz, V.; Hausicke, M.; Schmidt, A.-M.: "Scenario development without probabilities - focusing on the most important scenario". In: European Journal of Futures Research 2 (2014) 1,

[10] Reibnitz, U. v.: "Szenario-Technik. Instrumente für die unternehmerische und persönliche Erfolgsplanung". Gabler, Wiesbaden, 1992.

[11] Götze, U.: "Szenario-Technik in der strategischen Unternehmensplanung". Dt. Univ.-Verl. [u.a.], Wiesbaden, 1993.

[12] Gausemeier, J.; Fink, A.; Schlake, O.: "Szenario-Management. Planen und Führen mit Szenarien". Hanser, München, Wien, 1996.

[13] Gräßler, I.; Pottebaum, J.; Scholle, P.: „Integrated Process and Data Model for Agile Strategic Planning". In: Vajna, S. (Hrsg.) 11 ${ }^{\text {th }}$ International Workshop on Integrated Design Engineering, 2017.

[14] Grienitz, V.; Schmidt, A.-M.: "Scenariobased Complexity Management by adapting the Methods of Social Network Analysis". In: Zinn, C. D.; Ferrer, J. (Hrsg.) Proceedings of the International Multi-Conference on Complexity, Informatics and Cybernetics: IMCIC, 2010, S. 61-66. 
[15] Arcade, J.; Godet, M.; Meunier, F.; Roubelat, F.: "Structural analysis with the MICMAC method \& Actors' strategy with the MACTOR method". In: Futures Research Methodology, The Millennium Project, 1999 , S. 1-69.

[16] Kosow, H.: "New outlooks in traceability and consistency of integrated scenarios". In: European Journal of Futures Research 3 (2015) 1, S. 16.

[17] Grienitz, V.; Schmidt, A.-M.: „Weiterentwicklung der Konsistenzanalyse auf Basis evolutionärer Strategien für die Entwicklung von Markt- und Umfeldszenarien". In: Gausemeier, J. (Hrsg.) Vorausschau und Technologieplanung. Paderborn, 2009, S. 409-433.

[18] Hofmeister, P.: "Evolutionäre Szenarien. Dynamische Konstruktion alternativer Zukunftsbilder mit unscharfen Regelbasen". Kovac, 2000.

[19] Nitzsch, R. von; Weber, M.; Wietheger, D.: "KONMACA - Ein Programmsystem zur Unterstützung der Szenarioanalyse". In: Arbeitsberichte des Instituts für Wirtschaftswissenschaften (1985) 3, S. 1-56.

[20] Pottebaum, J.; Gräßler, I.: „Reliable Input for Strategic Planning: The Integrated Scenario Data Model". In: Villmer, F.-J.; Padoanao, E. (Hrsg.) Proceedings of $6^{\text {th }}$ International Conference Production Engineering and Management, 2016, S. 99-110.

[21] Gräßler, I.; Pottebaum, J.; Scholle, P.: "Influence Factors for Innovation in Digital Self-Preparedness Services and Tools". In: International Journal of Information Systems for Crisis Response and Management 10 (2018) 1, S. 20-37.

[22] Tapinos, E.: "Scenario planning at business unit level". In: Futures 47 (2013), S. 17-27.

[23] Millett, S. M.: "The future of scenarios. Challenges and opportunities". In: Strategy \& Leadership 31 (2003) 2, S. 16-24.

[24] Blessing, Lucienne T. M.; Chakrabarti, A.: "DRM, a Design Research Methodology". Springer London, Guildford, Surrey, 2009.

[25] Brin, S.; Page, L.: "The anatomy of a large-scale hypertextual Web search engine". In: Computer Networks and ISDN Systems 30 (1998) 1-7, S. 107-117.

[26] Bryan, K.; Leise, T.: "The $\$ 25,000,000,000$ Eigenvector. The Linear Algebra behind Google". In: SIAM Review 48 (2006) 3, S. 569-581.

[27] Fu, H.-H.; Lin, D. K. J.; Tsai, H.-T.: "Damping factor in Google page ranking". In: Applied Stochastic Models in Business and Industry 22 (2006) 5-6, S. 431-444.

[28] Gräßler, I.; Thiele, H.; Oleff, C.; Scholle, P.; Schulze, V.: "Method for Analysing Requirement Change Propagation Based on a Modified Pagerank Algorithm". In: Design Society (Hrsg.) International Conference on Engineering Design 2019, 2019. 